\title{
THE LACK OF TORUS EMISSION FROM BL LACERTAE OBJECTS: AN INFRARED VIEW OF UNIFICATION WITH WISE
}

\author{
Richard M. Plotkin ${ }^{1}$, Scott F. Anderson ${ }^{2}$, W. N. Brandt ${ }^{3,4}$, Sera Markoff $^{1}$, Ohad Shemmer $^{5}$, And Jianfeng Wu $^{3,4}$ \\ ${ }^{1}$ Astronomical Institute “Anton Pannekoek," University of Amsterdam, Science Park 904, 1098 XH, Amsterdam, The Netherlands; r.m.plotkin@uva.nl \\ ${ }^{2}$ Department of Astronomy, University of Washington, Box 351580, Seattle, WA 98195, USA \\ ${ }^{3}$ Department of Astronomy and Astrophysics, Pennsylvania Sate University, 525 Davey Laboratory, University Park, PA 16802, USA \\ ${ }^{4}$ Institute for Gravitation and the Cosmos, Pennsylvania State University, University Park, PA 16802, USA \\ ${ }^{5}$ Department of Physics, University of North Texas, Denton, TX 76203, USA \\ Received 2011 October 19; accepted 2011 December 21; published 2012 January 11
}

\begin{abstract}
We use data from the Wide-Field Infrared Survey Explorer (WISE) to perform a statistical study on the mid-infrared (IR) properties of a large number $\left(\sim 10^{2}\right)$ of BL Lac objects-low-luminosity active galactic nuclei (AGNs) with a jet beamed toward the Earth. As expected, many BL Lac objects are so highly beamed that their jet synchrotron emission dominates their IR spectral energy distributions. In other BL Lac objects, however, the jet is not strong enough to completely dilute the rest of the AGN emission. We do not see observational signatures of the dusty torus from these weakly beamed BL Lac objects. The lack of observable torus emission is consistent with suggestions that BL Lac objects are fed by radiatively inefficient accretion disks. Implications for the "nature versus nurture" debate for FR I and FR II radio galaxies are briefly discussed. Our study supports the notion that, beyond orientation, accretion rate plays an important role in AGN unification.
\end{abstract}

Key words: accretion, accretion disks - BL Lacertae objects: general - infrared: galaxies

Online-only material: color figures

\section{INTRODUCTION}

The standard orientation-based unification paradigm posits that every active galactic nucleus (AGN) is comprised of the same basic components: an accretion disk around a supermassive black hole, a broad emission line region (BELR), an obscuring torus, and a narrow-line region; radio-loud AGNs also launch large-scale relativistic jets (e.g., Antonucci 1993; Urry \& Padovani 1995). The torus is a key component in this unified model, as it can block the BELR along certain lines of sight, and its dust also reprocesses UV/X-ray radiation into the infrared (IR). In simple unification, the only other way to hide the BELR is also via a geometric argument: emission lines are outshone by a relativistic jet beamed toward Earth.

In addition to orientation, intrinsic differences among AGNs also play a role in AGN unification. For example, Urry \& Padovani (1995) conclude their seminal review with 10 outstanding questions, including one regarding Fanaroff-Riley galaxies (FR; Fanaroff \& Riley 1974): "do FR Is have BELRs?" The significance of this question is that if FR I galaxies lack BELRs, then intrinsic properties might play a prominent role in driving the so-called FR I/II dichotomy (in which the more powerful FR II galaxies have edge-brightened radio lobes). Indications so far are that emission line luminosities from FR I galaxies are 5-30 times weaker than for FR II galaxies, and FR I galaxies may also have weaker tori (Zirbel \& Baum 1995; Chiaberge et al. 1999; Donato et al. 2004; although also see Cao \& Rawlings 2004; Leipski et al. 2009). However, the requisite observations and their interpretation are technically challenging (Capetti et al. 2000; Evans et al. 2006), and there are still many ongoing "nature versus nurture" debates.

We can turn to beamed versions of FR I and FR II galaxies for additional guidance-i.e., BL Lac objects and flat spectrum radio quasars (FSRQs), respectively, which are collectively called blazars. Broad emission lines and sometimes dusty tori are routinely detected in the spectral energy distributions (SEDs) of FSRQs, but less often from BL Lac objects (e.g., Abdo et al. 2010; Giommi et al. 2011). However, it is generally still not observationally clear if BL Lac BELRs and tori appear weak solely due to dilution by jet emission (e.g., Chen \& Shan 2011; Malmrose et al. 2011), or rather if they really are intrinsically weak (e.g., Ghisellini et al. 2011; Sbarrato et al. 2011). Constraints on blazar BELRs and tori are furthermore important, as both components are potential sources of seed photons for Comptonized gamma-ray emission, especially for FSRQs and low synchrotron peaked (LSP) BL Lac objects. Note, gamma-ray emission from high synchrotron peaked (HSP) BL Lac objects is generally consistent with synchrotron selfCompton (e.g., Abdo et al. 2010).

The preliminary data release of the Wide-field Infrared Survey Explorer (WISE; Wright et al. 2010) recently opened a new multiwavelength window over a large area of the sky. In this Letter, we investigate the tori of low-luminosity radio galaxies by examining BL Lac objects detected by WISE. We describe our BL Lac sample in Section 2. In Section 3 we describe their IR properties, and results are discussed in Section 4. All spectral indices are defined as $f_{v} \propto v^{-\alpha_{v}}$, and we define the broadband radio to IR spectral index $\alpha_{r i}=-\log \left(L_{r} / L_{i}\right) / 4.25$, where $L_{r}$ and $L_{i}$ are monochromatic luminosities at $5 \mathrm{GHz}$ and $3.4 \mu \mathrm{m}$ rest frame, respectively. We adopt the following cosmology: $H_{0}=71 \mathrm{~km} \mathrm{~s}^{-1} \mathrm{Mpc}^{-1} ; \Omega_{M}=0.27$; and $\Omega_{\Lambda}=0.73$.

\section{THE BL LAC SAMPLE}

We start with 590 confirmed radio-loud BL Lac objects from the catalog of Plotkin et al. (2010, hereafter P10) ${ }^{6}$ which were selected from Sloan Digital Sky Survey (SDSS) spectroscopy

\footnotetext{
6 A subset of radio-quiet BL Lac candidates from P10 with WISE coverage is discussed in Wu et al. (2011). Wu et al. (2011) show that many are likely low-redshift analogs to weak line quasars (WLQs), which are high-redshift $(z>3)$ quasars with unusually weak or missing BELRs but normal dusty tori (see Lane et al. 2011).
} 


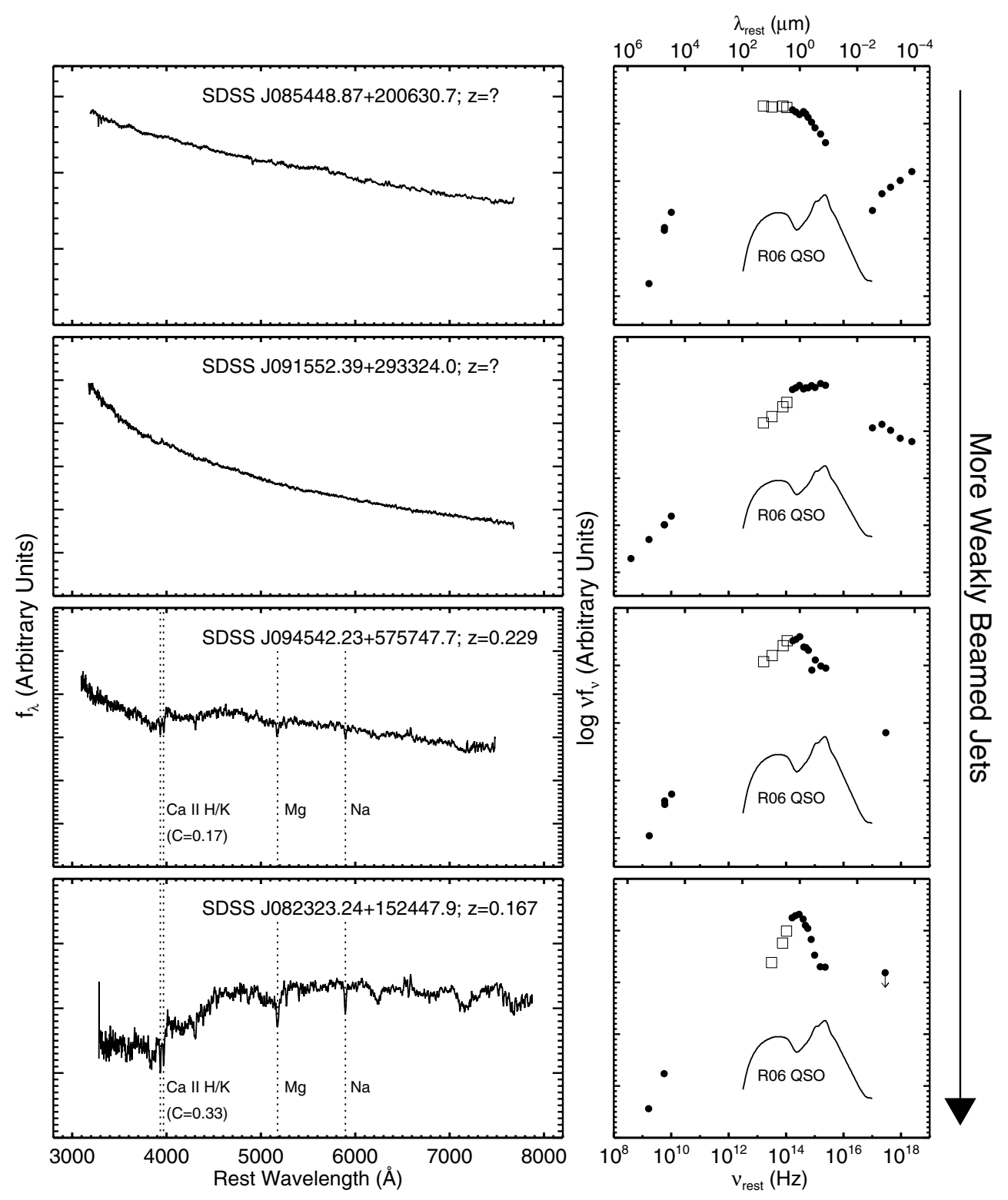

Figure 1. Sample SDSS optical spectra for four BL Lac objects (left) and corresponding SEDs (right). For illustrative purposes, we assume $z=0.2$ for the top two rows. WISE data points are shown as open squares, and the other SED multiwavelength data are assembled as in Section 4.3.5 of Plotkin et al. (2012). For comparison, we include a typical quasar SED (from Richards et al. 2006, R06), which shows thermal torus dust emission peaking redward of $1-2 \mu \mathrm{m}\left(3.0-1.5 \times 10^{14} \mathrm{~Hz}\right.$ ).

(York et al. 2000). P10 require no SDSS spectrum to show an emission feature with REW > $5 \AA$, and the Ca II $\mathrm{H} / \mathrm{K}$ break, $C$, must be smaller than $40 \%$ (e.g., Stocke et al. 1991; Marchã et al. 1996). The H/K break (i.e., the fractional change of continuum flux surrounding $4000 \AA$ rest frame) quantifies the host galaxy (HG) contribution to each SDSS spectrum. A spectrum with a more highly beamed jet will show smaller $C$ because the jet is brighter compared to the (unbeamed) HG (see, e.g., Landt et al. 2002). Of the $\sim 60 \%$ of BL Lac objects for which P10 can determine redshifts, most (and essentially all at $z<0.5$ ) are derived via absorption lines from $\mathrm{HG}$ starlight and are thus among the most weakly beamed P10 BL Lac objects (see Figure 1).

We correlate the P10 BL Lac objects to the preliminary WISE data release using a $3^{\prime \prime}$ search radius. We require WISE detections with signal-to-noise ratio $(\mathrm{S} / \mathrm{N})>3$ in the $\mathrm{W} 1[3.4 \mu \mathrm{m}]$, $\mathrm{W} 2[4.6 \mu \mathrm{m}]$, and $\mathrm{W} 3[12 \mu \mathrm{m}]$ bands. Requiring $\mathrm{S} / \mathrm{N}>3$ for the $\mathrm{W} 4[22 \mu \mathrm{m}]$ band would reduce our sample by at least a factor of two. We retain 157 objects (see Figure 1). To create comparison samples of normal quasars and early-type galaxies (ETGs; BL Lac objects live perhaps universally in large ellipticals; Urry et al. 2000), we also correlate 105,783 spectroscopically confirmed Type I SDSS quasars (Schneider et al. 2010) and 8666 (inactive) early SDSS galaxies (Bernardi et al. 2003 ) to WISE, applying the same $\mathrm{S} / \mathrm{N}$ constraint as above. All P10 BL Lac objects have $z<2$, so we also restrict the comparison quasars to $z<2$. We further consider only quasars with central black hole masses $10^{8}<M_{\mathrm{bh}} / M_{\odot}<10^{9}$ (masses from Shen et al. 2011), and ETGs with stellar velocity dispersions $200 \mathrm{~km} \mathrm{~s}^{-1}<\sigma_{\text {disp }}<320 \mathrm{~km} \mathrm{~s}^{-1}$. The above values of $M_{\mathrm{bh}}$ and $\sigma_{\text {disp }}$ are typical for BL Lac HGs (see León-Tavares et al. 2011; Plotkin et al. 2011). We are left with 13,881 quasars and 747 ETGs. Throughout, we convert WISE magnitudes to flux densities $\left(f_{v}\right)$ using the published (iso) zero points. ${ }^{7}$

\footnotetext{
7 http://wise2.ipac.caltech.edu/docs/release/prelim/expsup/figures/ sec4_3gt4.gif.
} 


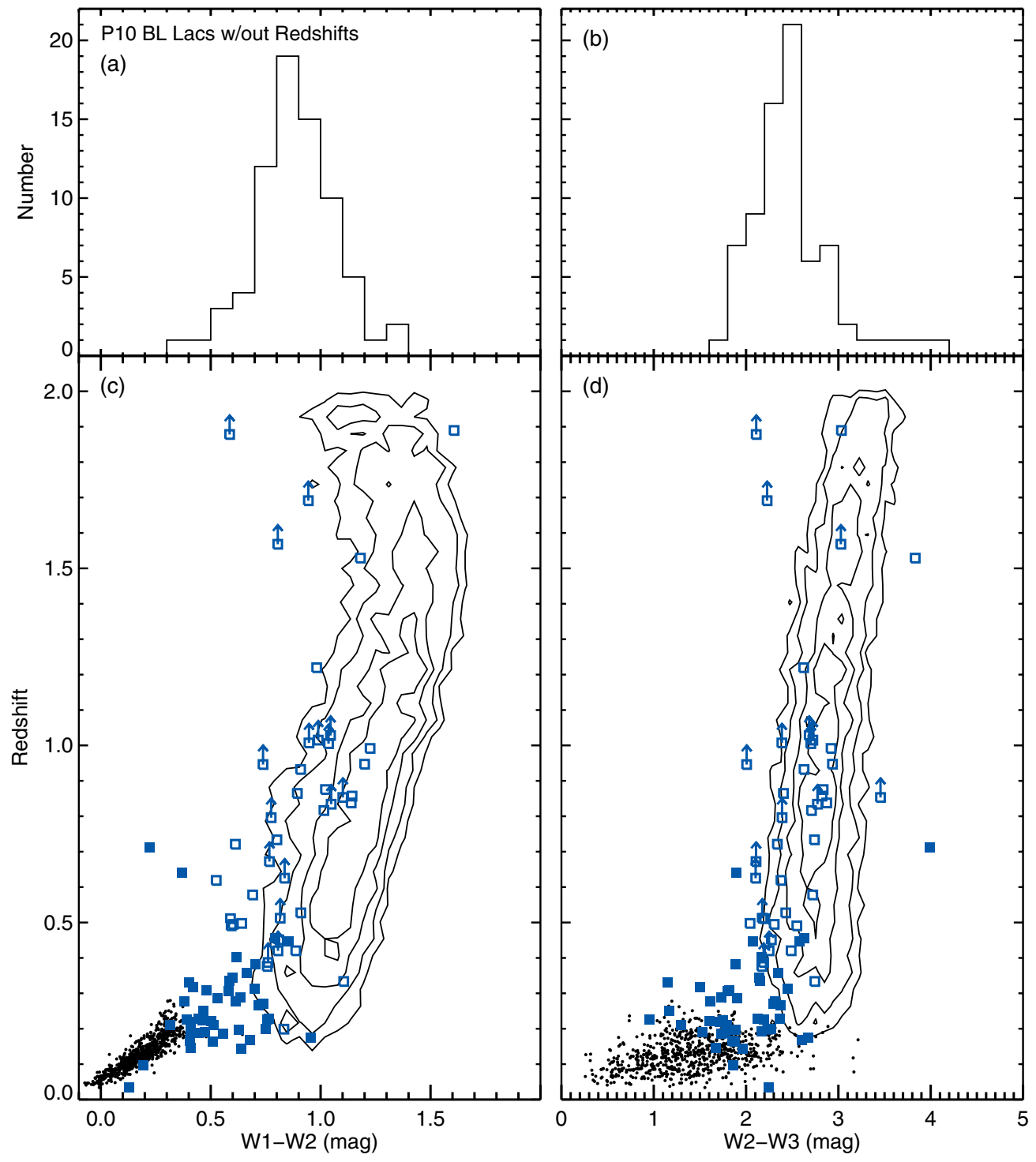

Figure 2. Distributions of W1-W2 (a) and W2-W3 (b) for P10 objects lacking redshifts. Panels (c) and (d) show redshift vs. IR colors for the remaining P10 BL Lac objects (blue squares). Open symbols indicate less reliable redshifts. We also show the IR colors of our comparison quasars (contours) and ETGs (filled circles).

(A color version of this figure is available in the online journal.)

\section{BL LAC INFRARED PROPERTIES}

The WISE colors, W1-W2 and W2-W3, for the P10 sample and for our comparison quasars and elliptical galaxies are shown in Figure 2 as a function of redshift. The dusty torus of quasars makes their IR colors redder than for ETGs. The first-order dependence of the quasar IR colors on redshift is simply due to the torus being redshifted through the WISE filters. Although some BL Lac objects have WISE colors similar to quasars, BL Lac objects tend to populate the bluer edge of the quasars' color space. For most lower-redshift BL Lac objects, this is because of HG contamination (elliptical galaxy SEDs emit most of their radiation in the near-IR and appear blue in the midIR; see Figure 1(d)). However, beamed synchrotron emission should completely dominate the IR emission from the higher redshift objects and those lacking redshifts (see, e.g., Padovani et al. 2006; Chen \& Shan 2011). We thus expect any overlap between the IR colors of highly beamed BL Lac objects and SDSS quasars to be coincidental, since different mechanisms (i.e., synchrotron versus thermal dust emission) are producing their IR flux.

\subsection{Constraints on BL Lac Dusty Tori}

BL Lac objects bridge the IR color space between normal galaxies and quasars (Figure 3(a)). One might expect the most weakly beamed BL Lac objects to show contributions from both HG starlight and from thermal dust emission from the torus. Then, the most weakly beamed BL Lac objects would look more similar to quasars that appear extended in their SDSS images (red plus signs). However, the weakly beamed BL Lac objects' IR colors are too blue for a significant contribution from the torus. In this section we determine if the lack of torus signatures is simply because even weakly beamed jets (and HGs) can still outshine the torus.

All ETGs in our comparison sample have $z<0.3$, so we restrict ourselves to $28 \mathrm{BL}$ Lac objects with $z<0.3$ in this section. All 28 objects appear as point sources in their WISE images. There is thus little concern that the WISE photometry includes different fractions of the total HG flux as a function of redshift. We also verify that they show no significant correlation between redshift and either WISE color. All 28 objects have $z>0.1$, so, for uniform comparison, we only consider ETGs 

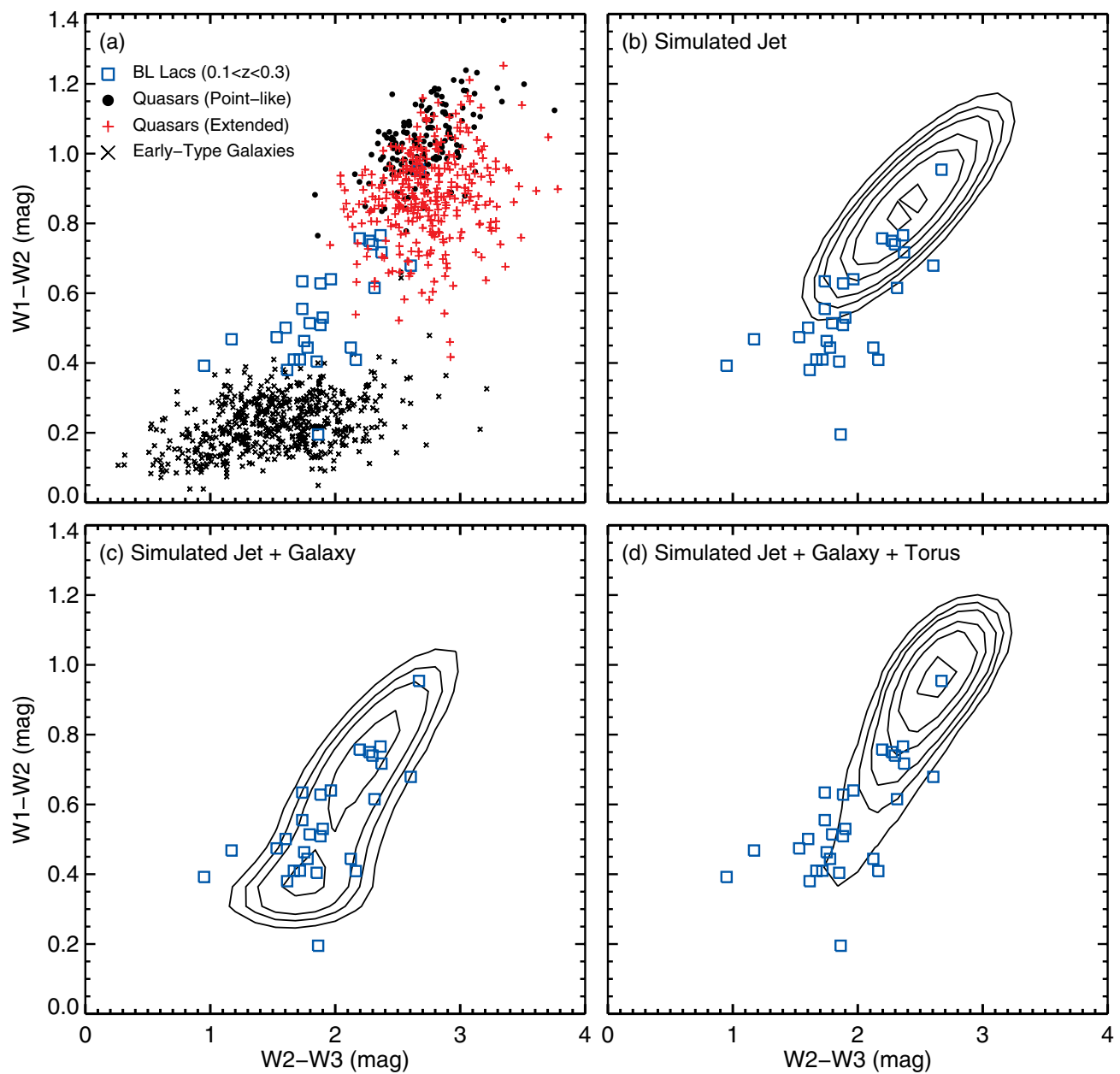

Figure 3. (a) Color-color diagram for 28 low-redshift BL Lac objects (blue squares), 150 comparison quasars with SDSS point-like morphologies (circles), 320 quasars that appear extended in SDSS imaging, and 530 ETGs (crosses). In the remaining panels, contours show expected BL Lac colors for MC simulations including only (b) a beamed jet, (c) a jet and host galaxy, and (d) a jet, host galaxy, and torus (see the text for details). Quasars and ETGs are omitted from panels (b)-(d) for clarity. (A color version of this figure is available in the online journal.)

and quasars from $0.1<z<0.3$ (530 and 470 objects, respectively).

There are three emission components that must be modeled in the IR: (1) starlight from the HG; (2) beamed jet synchrotron emission; and (3) dusty torus emission. To test if the dusty torus is present, we run Monte Carlo (MC) simulations. These simulations involve building a set of probability density functions (pdfs; see Sections 3.1.1-3.1.3), from which we take $10^{6}$ random draws to estimate each IR-emitting component's monochromatic luminosity in each WISE filter. From these luminosities we synthesize the expected WISE color space occupied by BL Lac populations with and without the torus. Throughout we estimate all luminosities at $z=0.2$ to minimize $K$-correction uncertainties and restrict comparison samples to a narrow redshift range near $z=0.2$. For all pdfs modeled as $(\log )$ normal distributions below, we first check that their observed parameter distributions are indeed approximately Gaussian.

\subsubsection{IR Emission from Host Galaxy Starlight}

We consider the observed properties of 164 ETGs with $z=0.22 \pm 0.05$ (the median of our low-redshift BL Lac sample). We first build a pdf for the monochromatic luminosity in the W2 filter, assuming a lognormal distribution with $\left\langle\log L_{v}\right\rangle_{h g, w 2}=29.82 \pm 0.14 \mathrm{erg} \mathrm{s}^{-1} \mathrm{~Hz}^{-1}$. Then we make pdfs for HG colors using normal distributions with $\langle W 1-W 2\rangle=$
$0.30 \pm 0.066 \mathrm{mag}$ and $\langle W 2-W 3\rangle=1.58 \pm 0.43 \mathrm{mag}$. Randomly drawing from each distribution allows us to estimate $\mathrm{HG}$ monochromatic luminosities in each WISE filter. This method implicitly accounts for measurement uncertainties.

\subsubsection{Jet Synchrotron in the IR}

We begin with an unbeamed $5 \mathrm{GHz}$ radio core luminosity $\left(v L_{v}\right)_{j, r}$ and use the broadband radio-IR spectral index $\alpha_{r i}$ to estimate an unbeamed IR jet luminosity in the W1 filter, $\left(v L_{v}\right)_{j, w 1}$. Beamed IR jet luminosity is then calculated as $\left(v L_{v}\right)_{j, w 1}^{\prime}=\left(v L_{v}\right)_{j, w 1} \delta^{2+\alpha_{\text {wise }}}$ (see Lind \& Blandford 1985), where $\delta=[\gamma(1-\beta \cos \theta)]^{-1}$ is the Doppler parameter, $\gamma=$ $\left(1-\beta^{2}\right)^{-0.5}, \theta$ is the viewing angle $\theta=0$ corresponds to a perfectly aligned jet), and $\beta$ is the jet velocity normalized to the speed of light. We then use $\left(v L_{v}\right)_{j, w 1}^{\prime}$ and the local IR spectral index $\alpha_{\text {wise }}$ to estimate beamed jet luminosities in the W2 and W3 filters. ${ }^{8}$

To estimate beamed jet luminosities for a random viewing angle, Lorentz factor, and jet power, we randomly draw from the following pdfs: a lognormal distribution for $\left(v L_{v}\right)_{j, r}$ with

\footnotetext{
8 We assume that $\alpha_{\text {wise }}$ and $\alpha_{r i}$ are not strongly affected by beaming, which is not strictly true but is a reasonable approximation once the jet is highly beamed enough to appear as a BL Lac object.
} 
$\left\langle\log v L_{v}\right\rangle_{j, r}=40.77 \pm 0.69 \mathrm{erg} \mathrm{s}^{-1}$ (derived from Equation (2) and Table 4 of $\mathrm{Wu}$ et al. 2007), a normal distribution for $\gamma$ with $\langle\gamma\rangle=7 \pm 0.7$ (e.g., see Merloni \& Heinz 2007), and a uniformly distributed $\theta$ from $0-40^{\circ}$. For $\alpha_{\text {wise }}$, we fit power laws to the $\mathrm{W} 1, \mathrm{~W} 2$, and $\mathrm{W} 3$ flux densities (in $\log f_{v}-\log v$ ) for the $69 \mathrm{P} 10 \mathrm{BL}$ Lac objects lacking redshifts. Based on those measurements, we then randomly draw $\alpha_{\text {wise }}$ from a normal distribution with $\left\langle\alpha_{\text {wise }}\right\rangle=0.60 \pm 0.43$. We similarly measure $\alpha_{r i}$ for those $69 \mathrm{BL}$ Lac objects (assuming $z=0.2$ ) using the radio fluxes in $\mathrm{P} 10$ and the $\mathrm{W} 1$ filter. We then randomly draw $\alpha_{r i}$ from a normal distribution with $\left\langle\alpha_{r i}\right\rangle=0.40 \pm 0.11$. Finally, once we estimate luminosities in each filter as described in the previous paragraph, we add random noise to each luminosity by assuming $\sigma_{L} / L= \pm 0.05,0.05$, and 0.1 in the $\mathrm{W} 1, \mathrm{~W} 2$, and $\mathrm{W} 3$ filters, respectively (based on typical WISE flux measurement uncertainties for our full BL Lac sample).

\subsubsection{IR Emission from a "Typical" Obscuring Torus}

We employ a realistic coupling between the jet and accretion flow to estimate IR luminosities from the dusty torus. First, we estimate X-ray luminosity $L_{x}$ (from 2 to $10 \mathrm{keV}$ ) using $\left(v L_{v}\right)_{j, r}$ from above and the following relations from Merloni \& Heinz (2007), based on a sample of low-luminosity AGNs with mechanical jet power estimates:

$$
\begin{aligned}
\log \left(\frac{L_{\text {kin }}}{L_{\text {Edd }}}\right)= & (0.49 \pm 0.07)\left(\log \left[\frac{L_{x}}{L_{\text {Edd }}}\right]-\log [B / 0.2]\right) \\
& -(0.78 \pm 0.36)
\end{aligned}
$$

and

$$
\log L_{\mathrm{kin}}=(0.81 \pm 0.11) \log \left(v L_{v}\right)_{j, r}+11.9_{-4.4}^{+4.1},
$$

where $L_{\text {kin }}$ is the kinetic power of the jet in erg s $\mathrm{s}^{-1}, L_{\mathrm{Edd}}=$ $1.3 \times 10^{38}\left(M / M_{\odot}\right) \mathrm{erg} \mathrm{s}^{-1}$ is the Eddington luminosity, and $B$ is fraction of the bolometric luminosity emitted from 2 to $10 \mathrm{keV}$. We note that Equations (1) and (2) are consistent with the theoretical scalings for jet dominated accretion flows, $\left(\left[v L_{v}\right]_{j, r} / L_{\text {edd }}\right) \propto\left(L_{\text {kin }} / L_{\text {Edd }}\right)^{17 / 12}$ and $\left(L_{\text {kin }} / L_{\text {Edd }}\right) \propto$ $\left(L_{x} / L_{\text {Edd }}\right)^{0.5}$ (e.g., Falcke \& Biermann 1995). The above scalings are applicable to hard state X-ray binaries and their supermassive analogs, including BL Lac objects (see, e.g., Plotkin et al. 2012).

We then assume that X-ray luminosity is a good tracer for the torus' IR luminosity, using the IR/X-ray correlation from Gandhi et al. (2009):

$$
\log \left(\frac{v L_{v}}{10^{43}}\right)_{12.3 \mu \mathrm{m}}=(1.11 \pm 0.07) \log \left(\frac{L_{x}}{10^{43}}\right)+(0.19 \pm 0.05),
$$

where $\left(\nu L_{v}\right)_{12.3 \mu \mathrm{m}}$ is the IR luminosity at $12.3 \mu \mathrm{m}$ and the X-ray luminosity, $L_{x}$, is from $2-10 \mathrm{keV}$.

Combining Equations (1)-(3) leaves us the following relation for the IR torus emission at $12.3 \mu \mathrm{m}$, assuming an unbeamed radio core luminosity at $5 \mathrm{GHz}$ :

$$
\begin{aligned}
\log \left(\frac{\nu L_{v}}{10^{43}}\right)_{12.3 \mu \mathrm{m}}= & 1.84 \log \left(\frac{\nu L_{v}}{10^{40}}\right)_{j, r} \\
& -1.16 \log \left(\frac{L_{\text {Edd }}}{3.78 \times 10^{46}}\right) \\
& +1.11 \log \left(\frac{B}{0.1}\right)+0.43
\end{aligned}
$$

where we assume $B=0.1$ (to conservatively err on the side of underestimating the torus flux). Finally, we extrapolate torus luminosity to the W2 filter assuming an IR spectral index of 0.15 between 12.3 and $4.6 \mu \mathrm{m}$ (estimated at $z=0.2$ from the average Richards et al. 2006 quasar SED), and we estimate IR luminosities in the W1 and W3 filters using pdfs based on the WISE colors of 150 quasars $(0.1<z<0.3)$ with point-like SDSS morphologies.

For the MC simulations, we build the following additional pdfs. For $L_{\text {Edd }}$ we assume that BL Lac black hole masses are lognormal with $\langle\log M\rangle=8.54 \pm 0.40 M_{\odot}$ (Plotkin et al. 2011). Since Equation (3) relies on several assumptions that propagate nonlinearly, we randomly add intrinsic scatter to each estimate of $\left(\log v L_{v}\right)_{12.3 \mu \mathrm{m}}$ assuming a generous $\sigma_{\text {int }}= \pm 1.5 \mathrm{dex}$. For the pdfs for $W 1-W 2$ and $W 2-W 3$, we first randomly draw $W 1-W 2$ from a normal distribution with $\langle W 1-W 2\rangle=$ $1.03 \pm 0.10 \mathrm{mag}$. Since quasar WISE colors are correlated, we then use the best-fit relation $W 2-W 3=1.19+1.47(W 1-W 2)$ to build the $W 2-W 3$ pdf, and we add random noise using the root-mean-square (rms) scatter about the best-fit regression, $\sigma_{\text {rms }} \pm 0.23$ mag.

\subsubsection{Lack of Observational Signatures from the Dusty Torus}

We perform $10^{6} \mathrm{MC}$ simulations to estimate luminosities from each IR-emitting component in each WISE filter. Figure 3 shows the synthesized WISE colors if only one, two, or all three components are included. Note that the simulated jets encompass a range of viewing angles $\theta<40^{\circ}$ and Lorentz factors. Massaro et al. (2011) show that blazars fall in a distinct region of IR color space, which they call the "WISE blazar strip." The most highly beamed P10 BL Lac objects (i.e., the 69 lacking redshifts) also fall within the strip, and a Kolmogorov-Smirnov (K-S) test shows that the $\mathrm{W} 1-\mathrm{W} 2$ and $\mathrm{W} 2-\mathrm{W} 3$ distributions for those 69 BL Lacs and our synthesized "blazar strip" (panel b) are not significantly different $(p \sim 0.4$ and 0.6 , respectively). However, for the 28 weakly beamed objects, a bluer IR component (i.e., the HG) is necessary ( $p \sim 10^{-11}$ and $10^{-6}$ from K-S tests comparing their $\mathrm{W} 1-\mathrm{W} 2$ and $\mathrm{W} 2-\mathrm{W} 3$ distributions to the synthesized jet).

To explain the SDSS population of weakly beamed BL Lac objects (blue squares), we favor the synthesized colors including the jet and HG (Figure 3(c)) over the colors that also include the torus (Figure 3(d)). The simulations including the torus predict that BL Lac colors should be weighted toward redder IR colors, while the simulations with only the jet and galaxy predict IR colors more consistent with observations. K-S tests show that the $\mathrm{W} 1-\mathrm{W} 2$ and $\mathrm{W} 2-\mathrm{W} 3$ distributions for $28 \mathrm{BL}$ Lac objects with $0.1<z<0.3$ and the synthesized colors including the torus are statistically different ( $p \sim 10^{-11}$ and $10^{-8}$, respectively), while similar parent distributions are not highly excluded between the observed colors and the synthesized colors without the torus ( $p \sim 0.05$ and 0.02).

\section{DISCUSSION AND CONCLUSIONS}

We conclude from the above that our weakly beamed BL Lac objects lack observational signatures of the dusty torus in the mid-IR. Thus, BL Lac dusty tori appear weaker than the tori of luminous quasars (i.e., one cannot simply scale a normal quasar torus down to BL Lac luminosities). Another way to express this conclusion is that $\mathrm{BL}$ Lac tori have different properties than the tori of normal quasars. It is reasonable to extrapolate this result to highly beamed BL Lac objects, if 
beaming is primarily a geometric argument. If one interprets the torus as an extension of the BELR (e.g., Elitzur \& Shlosman 2006), then our study suggests that BL Lac BELRs are also intrinsically weak. We stress that this is a statistical conclusion. The simulations including the torus do predict a small population of weakly beamed BL Lac objects with WISE colors matching the observations, but that population is not large enough to explain the IR colors for the BL Lac population as a whole. However, we cannot exclude the presence of the torus/BELR from every single BL Lac object. Indeed, some BL Lac objects can show very weak broad emission lines in their optical spectra (especially some LSPs).

One explanation for weaker BL Lac tori is that their physical properties (i.e., torus geometries, dust properties, ionizing SEDs, etc.) are different than for luminous quasars. Another interpretation is the same one invoked for optically dull AGNs and "naked" Seyfert galaxies (e.g., Hawkins 2004; Trump et al. 2011), as follows. Consider a scenario where the BELR and torus are fed by a radiatively driven disk wind (e.g., Murray \& Chiang 1998). At sufficiently low accretion rate (below a few percent $L / L_{\text {Edd }}$ ), the inner region of the accretion disk is replaced by a radiatively inefficient accretion flow (RIAF). The RIAF is then no longer able to support a sufficient wind to populate the BELR and torus (e.g., Nicastro 2000). In this scenario, the strength of the BELR and torus is intimately connected to the efficiency of the accretion flow and therefore accretion rate. ${ }^{9}$ Indeed, such an "accretion rate dichotomy" has already been suggested to explain the BL Lac/FSRQ divide (with BL Lac objects fed by RIAFs; e.g., Böttcher \& Dermer 2002; Ghisellini et al. 2009). Such a scheme may also be responsible for the FR I/II divide (e.g., Ghisellini \& Celotti 2001; Wold et al. 2007). In fact, an important (supporting) point is that the classification of unbeamed radio galaxies based on high-excitation versus low-excitation emission lines (which are likely connected to the accretion flow) is more physically meaningful than the older FR I/II scheme based on radio morphology/jet power (see, e.g., Jackson \& Wall 1999; Hardcastle et al. 2009). Furthermore, such a division is naturally expected from the hysteresis displayed by accreting stellar mass black holes, where changes in X-ray spectral states for individual objects (i.e., at fixed orientations) may similarly be related to the efficiency of the inner accretion flow.

WISE offers a new perspective on the strength of the obscuring torus in low-luminosity radio galaxies. The WISE view of BL Lac objects is consistent with other (independent) indications of an accretion rate dichotomy among AGNs, adding a complementary piece to the puzzle. Further constraints on such a division in accretion mode is an important step for better understanding AGN unification.

We thank the anonymous referee. R.M.P. and S.M. acknowledge support from a Netherlands Organization for Scientific Research (NWO) Vidi Fellowship. W.N.B. and J.W. acknowledge support from NASA ADP grant NNX10AC99G. This publication makes use of data products from the Wide-Field Infrared Survey Explorer, which is a joint project of the University of California, Los Angeles, and the Jet Propulsion
Laboratory/California Institute of Technology, funded by the National Aeronautics and Space Administration.

\section{REFERENCES}

Abdo, A. A., Ackermann, M., Agudo, I., et al. 2010, ApJ, 716, 30

Antonucci, R. 1993, ARA\&A, 31, 473

Bernardi, M., Sheth, R. K., Annis, J., et al. 2003, AJ, 125, 1817

Böttcher, M., \& Dermer, C. D. 2002, ApJ, 564, 86

Cao, X., \& Rawlings, S. 2004, MNRAS, 349, 1419

Capetti, A., Schreier, E. J., Axon, D., et al. 2000, ApJ, 544, 269

Chen, P. S., \& Shan, H. G. 2011, ApJ, 732, 22

Chiaberge, M., Capetti, A., \& Celotti, A. 1999, A\&A, 349, 77

Donato, D., Sambruna, R. M., \& Gliozzi, M. 2004, ApJ, 617, 915

Elitzur, M., \& Shlosman, I. 2006, ApJ, 648, L101

Evans, D. A., Worrall, D. M., Hardcastle, M. J., Kraft, R. P., \& Birkinshaw, M 2006, ApJ, 642, 96

Falcke, H., \& Biermann, P. L. 1995, A\&A, 293, 665

Fanaroff, B. L., \& Riley, J. M. 1974, MNRAS, 167, 31

Gandhi, P., Horst, H., Smette, A., et al. 2009, A\&A, 502, 457

Ghisellini, G., \& Celotti, A. 2001, A\&A, 379, L1

Ghisellini, G., Maraschi, L., \& Tavecchio, F. 2009, MNRAS, 396, L105

Ghisellini, G., Tavecchio, F., Foschini, L., \& Ghirlanda, G. 2011, MNRAS, 414, 2674

Giommi, P., Padovani, P., Polenta, G., et al. 2011, MNRAS, in press (arXiv:1110.4706)

Hardcastle, M. J., Evans, D. A., \& Croston, J. H. 2009, MNRAS, 396, 1929

Hawkins, M. R. S. 2004, A\&A, 424, 519

Jackson, C. A., \& Wall, J. V. 1999, MNRAS, 304, 160

Landt, H., Padovani, P., \& Giommi, P. 2002, MNRAS, 336, 945

Lane, R. A., Shemmer, O., Diamond-Stanic, A. M., et al. 2011, ApJ, 743, 163

Leipski, C., Antonucci, R., Ogle, P., \& Whysong, D. 2009, ApJ, 701, 891

León-Tavares, J., Valtaoja, E., Chavushyan, V. H., et al. 2011, MNRAS, 411, 1127

Lind, K. R., \& Blandford, R. D. 1985, ApJ, 295, 358

Malmrose, M. P., Marscher, A. P., Jorstad, S. G., Nikutta, R., \& Elitzur, M. 2011, ApJ, 732, 116

Marchã, M. J. M., Browne, I. W. A., Impey, C. D., \& Smith, P. S. 1996, MNRAS, 281,425

Massaro, F., D’Abrusco, R., Ajello, M., Grindlay, J. E., \& Smith, H. A. 2011, ApJ, 740, L48

Merloni, A., \& Heinz, S. 2007, MNRAS, 381, 589

Murray, N., \& Chiang, J. 1998, ApJ, 494, 125

Nicastro, F. 2000, ApJ, 530, L65

Padovani, P., Giommi, P., Ábrahám, P., Csizmadia, S., \& Moór, A. 2006, A\&A, 456, 131

Plotkin, R. M., Anderson, S. F., Brandt, W. N., et al. 2010, AJ, 139, 390 (P10)

Plotkin, R. M., Markoff, S., Kelly, B. C., Körding, E., \& Anderson, S. F. 2012, MNRAS, 419, 267

Plotkin, R. M., Markoff, S., Trager, S. C., \& Anderson, S. F. 2011, MNRAS, 413,805

Richards, G. T., Lacy, M., Storrie-Lombardi, L. J., et al. 2006, ApJS, 166, 470

Sbarrato, T., Ghisellini, G., Maraschi, L., \& Colpi, M. 2011, MNRAS, submitted (arXiv:1108.0927)

Schneider, D. P., Richards, G. T., Hall, P. B., et al. 2010, AJ, 139, 2360

Shen, Y., Richards, G. T., Strauss, M. A., et al. 2011, ApJS, 194, 45

Stocke, J. T., Morris, S. L., Gioia, I. M., et al. 1991, ApJS, 76, 813

Trump, J. R., Impey, C. D., Kelly, B. C., et al. 2011, ApJ, 733, 60

Urry, C. M., \& Padovani, P. 1995, PASP, 107, 803

Urry, C. M., Scarpa, R., O’Dowd, M., et al. 2000, ApJ, 532, 816

Wold, M., Lacy, M., \& Armus, L. 2007, A\&A, 470, 531

Wright, E. L., Eisenhardt, P. R. M., Mainzer, A. K., et al. 2010, AJ, 140, 1868

Wu, J., Brandt, W. N., Anderson, S. F., et al. 2011, ApJ, in press (arXiv:1112.2228)

Wu, Z., Jiang, D. R., Gu, M., \& Liu, Y. 2007, A\&A, 466, 63

York, D. G., Adelman, J., Anderson, J., et al. 2000, AJ, 120, 1579

Zirbel, E. L., \& Baum, S. A. 1995, ApJ, 448, 521 